\title{
Sepsis induces early phrenic nerve neuropathy in rats
}

\author{
A. Nayci*, S. Atis", U. Comelekoglu", A. Ozge ${ }^{+}$, O. Ogenler", \\ B. Coskun ${ }^{\S}$ and S. Zorludemir ${ }^{\dagger}$
}

ABSTRACT: The aim of the present study was to investigate the electrophysiology of the phrenic nerve and the diaphragm muscle during sepsis.

In total, 26 rats underwent either sham laparotomy or caecal ligation and puncture (CLP). Electrophysiology was evaluated via a phrenic nerve conduction study and needle electromyography of the diaphragm, prior to CLP, 6 and $24 \mathrm{~h}$ post-CLP and on day 7. The histopathology of the diaphragm muscle and phrenic nerve was also examined on day 7.

In the sepsis group, the phrenic nerve conduction study showed decreased amplitude of compound action potential (CMAP), and prolongation in the duration and the latency of CMAP. The diaphragmatic needle electromyography showed decreased amplitude and frequency of the motor unit action potential (MUP), and prolongation in the duration of MUP, at all time points, compared with the pre-CLP values. The electrophysiological abnormalities were consistent with axonal and demyelinating phrenic nerve neuropathy. Electrophysiological abnormalities were present at $6 \mathrm{~h}$ with worsening at $24 \mathrm{~h}$ and on day 7. Histopathological examination showed normal muscular fibres and focally slight myelin degenerations of the phrenic nerve fibres.

In conclusion, sepsis induced phrenic nerve neuropathy as early as the 6 th $h$ in rats.

KEYWORDS: Diaphragm, myopathy, neuropathy, phrenic nerve, respiratory failure, sepsis

epsis is a common cause of mortality in intensive care units (ICU) and, in many cases, respiratory failure is a significant contributor to this mortality [1, 2]. It is now accepted that respiratory failure is related to impairment in the gas exchange system and failure of the pump, the two main components of the pump being respiratory musculature and peripheral nerve innervations [3].

It is necessary to understand the pathogenesis of respiratory failure in sepsis to develop effective strategies for the prevention and treatment of this disorder. The vast majority of experimental sepsis models, demonstrating failure of the respiratory musculature, have been focused on the mechanical properties of the striated muscle of the diaphragm [4-6]. Only a few studies have investigated the electrophysiology of the diaphragm during sepsis. LEON et al. [7] found that impaired neuromuscular transmission, related to decreased resting membrane potential, was an important cause of diaphragm dysfunction in septic animals. LiN et al. [8] reported impaired diaphragm contractility in association with diaphragm sarcolemma injury, with attendant abnormalities of myofibre membrane electrophysiology during sepsis. Neither of these studies addressed whether sepsis directly affects the diaphragm muscle or phrenic nerve innervation.

The electrophysiology of the diaphragm during sepsis, with the exception of a few isolated reports, has not been fully elucidated and, as far as current literature shows, a time-course study has not been carried out. In this regard, the present study aimed to investigate the electrophysiology of the diaphragm from the onset to the late phases of sepsis. In particular, it aimed to observe whether the respiratory failure observed in sepsis resulted directly from the diaphragm muscle and/or phrenic nerve innervation.

\section{MATERIALS AND METHODS \\ Experimental design}

The experimental protocol was approved by the Animal Care and Use Committee of the University of Mersin (Mersin, Turkey). The rats had free access to standard laboratory diet and water, and were maintained according to the recommendations of the National Institute of Health's guidelines for the care and use of laboratory animals [9].

In total, 26 Wistar albino rats, weighing 250$300 \mathrm{~g}$, were used in the study. The rats were
AFFILIATIONS

*Depts of Pediatric Surgery,

\#Chest Diseases,

Biophysics,

${ }^{+}$Neurology,

${ }^{\S}$ Histology and Embriology, Mersin University, School of Medicine, and

${ }^{f}$ Dept of Pathology, Cukurova

University, School of Medicine,

Mersin, Turkey.

CORRESPONDENCE

A. Nayci

Inonu Mah. 1405 Sok. Murat Apt. 2/

11

33110, Mersin

Turkey

Fax: 903243288742

E-mail: anayci@mersin.edu.tr

Received:

September 242004

Accepted after revision:

June 132005 
assigned into one of two groups, sham $(n=10)$ or sepsis $(n=16)$.

\section{Protocol for the induction of sepsis}

Intra-abdominal sepsis was produced using the caecal ligation and puncture (CLP) procedure [10]. The rats were anesthetised with ketamine hydrochloride intramuscularly $(50 \mathrm{mg} \cdot \mathrm{kg}$ body weight $^{-1}$; Ketalar; Eczacibasi-WL, Istanbul, Turkey). A mid-line laparatomy was performed, and the caecum was ligated distal to the ileocaecal valve to ensure the intestinal continuity was preserved. The caecum was punctured in two locations on the anti-mesentheric surface of the caecum, using an 18-gauge needle. The caecum was gently compressed until faeces were extruded and then returned to the peritoneum. The incision was closed in two layers. Animals recovered from anesthesia and moved about freely in the cages. The sham group underwent only the laparatomy procedure.

\section{Electrophysiological procedure}

Electrophysiological measurements of the diaphragm and the phrenic nerve were recorded prior to the CLP procedure in order to obtain control values, and then after 6 and $24 \mathrm{~h}$ postCLP and on day 7 to evaluate the effect of sepsis.

\section{Phrenic nerve conduction study}

Electrophysiological recordings (Biopac-MP 100; Biopac, Santa Barbara, CA, USA) were performed as described previously [11]. The active and reference surface $\mathrm{Ag} / \mathrm{AgCl}$ disc electrodes were used to record the phrenic nerve compound action potential (CMAP) using the modified MACLEAN and MATTIONI [12] technique. The active electrode was placed on the xiphoid process, the reference electrode was placed on the ipsilateral eighth intercostal space at the costocondral junction and the ground electrode was placed on the sternum (Medelec, Oxford, UK). The phrenic nerve was stimulated at a supramaximal voltage by pressing deeply along the posterior margin of the sternocleidomastoid muscle at the level of the cricoid cartilage. The supramaximal stimulus consisted of a single square pulse (intensity $10 \mathrm{~V}$; duration $0.5 \mathrm{~ms}$ ). At regular intervals it was ensured that stimulation voltage remained supramaximal. The recorded signals were then rectified and electrically integrated. The sampling rate was chosen as 20,000 samples $\cdot \mathrm{s}^{-1}$. The amplitude from the baseline to the negative peak, the duration and the latency of CMAP were measured.

\section{Needle electromyography of the diaphragm}

The monopolar needle electrode (Biopac-EL; Biopac) was inserted under the lower costal margin between the anterior axillary and mid-clavicular line. The reference electrode was placed on the adjacent costal margin and the ground electrode was placed on the sternum (Medelec). Insertional activity, spontaneous activity (fibrillation potential, positive sharpwave and fasciculation), motor unit recruitment pattern, motor unit action potential (MUP) and morphology (peak-to-peak amplitude, total duration and MUP $n \cdot 10 \mathrm{~s}^{-1}$ ) were evaluated quantitatively in resting state [13, 14].

\section{Histopathological examination}

The diaphragmatic specimens were snap-frozen in isopentane, cooled in liquid nitrogen and stored at $-70^{\circ} \mathrm{C}$. Cryostatic sections were cut at $6 \mu \mathrm{m}$ and processed using conventional histological methods, including haematoxylin-eosin staining, Gomari's trichrome, periodic acid-Schiff, oil red O, cytochrome oxidase, succinate dehydrogenase $\mathrm{NADH}$, adenosine triphosphatase ( $\mathrm{pH}$ 9.8), acid phosphatase and nonspecific esterase. The slides were then examined using light microscopy.

The phrenic nerve specimens were immediately fixed in Karnovsky's fixative and post-fixed in $1 \%$ osmium tetroxide. Light microscopic analysis was performed using eponembedded $1-\mu \mathrm{m}$ sections stained with toluidine blue.

\section{Statistical analysis}

After documenting the normal distribution by KolmogorovSmirnov, data were presented as means $\pm \mathrm{SD}$, analysed using ANOVA for the two factors (group and time) repeated measures and the Tukey post hoc test. Statistical significance was accepted when p-values were $<0.05$.

\section{RESULTS}

In the sepsis group, the rats manifested malaise, fever, chills, piloerection, ocular exudates and diarrhoea. The mice in the sepsis group became progressively more lethargic and moved about less. The rats were also tachypnoeic, and some had apparent respiratory distress. One rat died in $<24 \mathrm{~h}$ and three rats died in $<7$ days after CLP, secondary to respiratory arrest. These animals were excluded from the study. When sacrificed 7 days after CLP, the peritoneal cavity typically contained 1$2 \mathrm{~mL}$ of cloudy fluid. The caecum was inflated and gangrenous, and the puncture holes were visible. The liver and kidneys were engorged with blood, but the bowel was not markedly altered. Each animal had an intra-abdominal abscess, which was walled off.

\section{Electrophysiology}

\section{Phrenic nerve conduction study}

In the sham group, the amplitudes, durations and latency of CMAP remained unchanged throughout the study.

In the sepsis group, the amplitudes of CMAP decreased significantly to $8.99 \pm 0.81,7.83 \pm 0.89$ and to $6.15 \pm 1.31 \mathrm{mV}$ (after 6 and $24 \mathrm{~h}$, and on day 7, respectively), compared with pre-operative values $(p=0.012, p=0.0001$ and $p=0.0001$, respectively; fig. 1a). The duration of CMAP steadily prolonged to $2.64 \pm 0.39,3.20 \pm 0.69$ and to $3.51 \pm 0.70 \mathrm{~ms}$ (after 6 and $24 \mathrm{~h}$, and on day 7 , respectively), compared with preoperative levels $(\mathrm{p}=0.006, \mathrm{p}=0.0001$ and $\mathrm{p}=0.0001$, respectively; fig. 1b). The latency of CMAP was significantly prolonged to $0.52 \pm 0.13,0.64 \pm 0.15$ and to $0.69 \pm 0.16 \mathrm{~ms}$ (after 6 and $24 \mathrm{~h}$, and on day 7, respectively), compared to preoperative levels $(p=0.0001$ at all time points; fig. 1c).

\section{Diaphragmatic needle electromyography}

In the sham group, the amplitudes, durations and the number of MUP remained unchanged throughout the time-course.

However, in the sepsis group, the amplitudes of MUP increased significantly to $2.59 \pm 0.57 \mathrm{mV}$ and continued to increase to $3.00 \pm 0.97$ and $3.24 \pm 1.05 \mathrm{mV}$ (after 6 and $24 \mathrm{~h}$, and on day 7 , respectively), without any significant accompanying spontaneous activity when compared with the pre-operative levels $(p=0.005, p=0.002$ and $p=0.001$, respectively; fig. 2a). The corresponding duration of MUP steadily prolonged to $13.32 \pm 1.73$ and $17.00 \pm 2.40 \mathrm{~ms}$ (after 6 and $24 \mathrm{~h}$, respectively) 

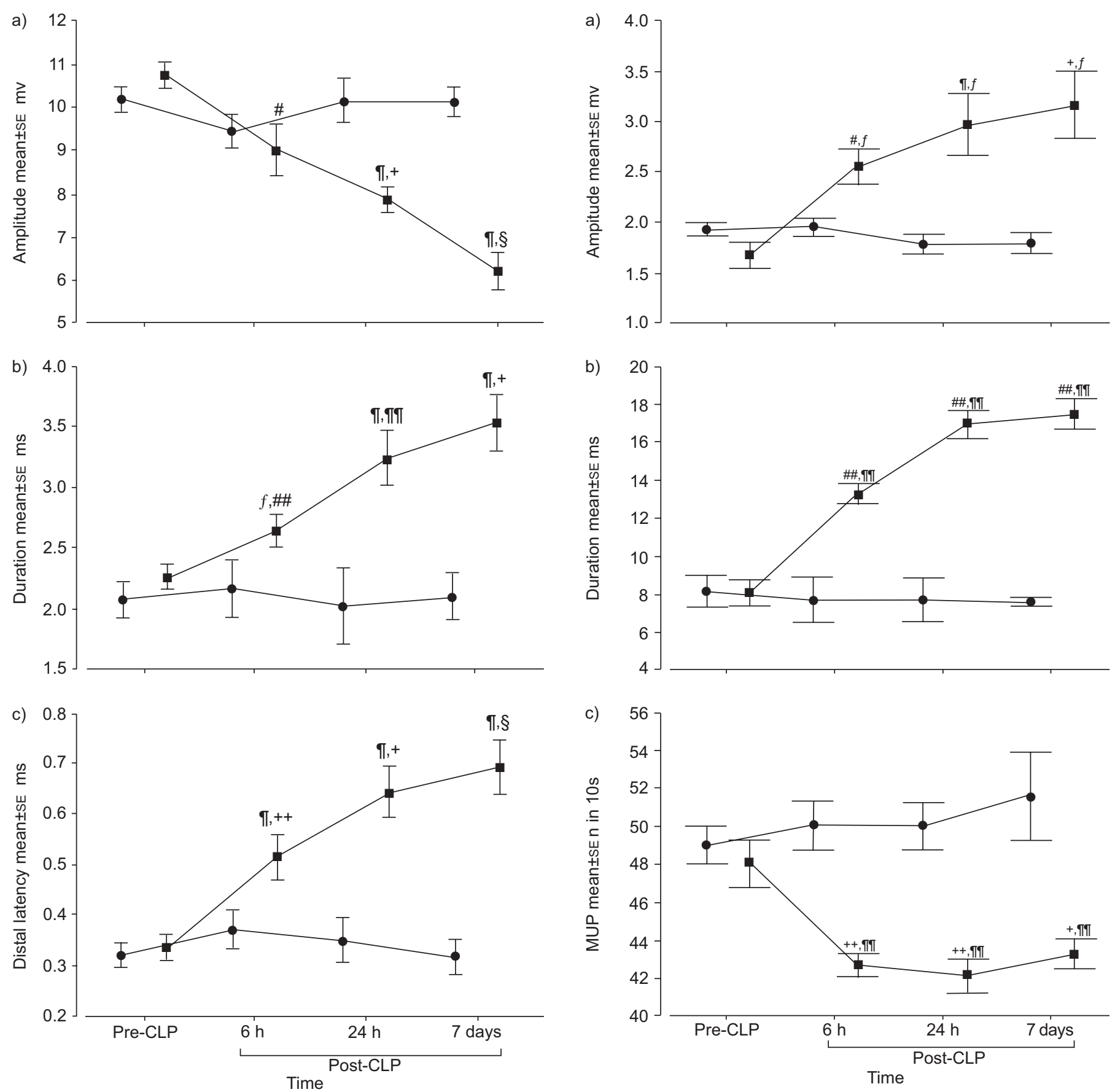

FIGURE 1. Compound action potential amplitudes of a) amplitude, b) duration

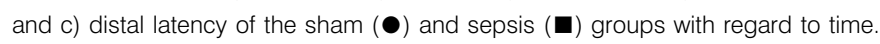
CLP: caecal ligation and puncture. ${ }^{\#}: p=0.012 ;{ }^{\natural}: p=0.0001{ }^{f}: p=0.006$ compared

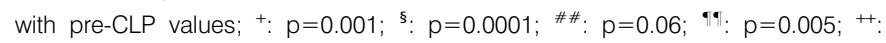
$p=0.037$ compared with the sham group.

and then drove to a plateau on day $7(17.58 \pm 2.43 \mathrm{~ms})$ when compared with the pre-operative levels $(\mathrm{p}=0.0001$ at all time points; fig. $2 \mathrm{~b}$ ). The number of the MUP was significantly decreased to $42.60 \pm 1.89$ after $6 \mathrm{~h}$, and then sustained up to $24 \mathrm{~h}(42.0 \pm 2.82)$ and on day $7(42.6 \pm 1.89)$, when compared with the pre-operative levels $(p=0.004, p=0.004$ and $p=0.001$,

FIGURE 2. Motor unit action potential amplitudes of a) amplitude, b) duration and c) number in a 10-s period of the sham $(\bullet)$ and sepsis ( $\mathbf{\square})$ groups with regard to time. CLP: caecal ligation and puncture. ${ }^{\#}: p=0.05 ; ": p=0.002 ;{ }^{+}: p=0.001 ;{ }^{\# \#}$ : $p=0.0001 ;{ }^{++}: p=0.004$ compared with the pre-CLP values; $\xi: p=0.028 ; f$ : $p=0.012 ; \%: p=0.0001$ compared with the sham group.

respectively; fig. 2c). The morphological changes in CMAP and MUP are shown in figure 3.

\section{Histopathology}

In the sham group, the diaphragm (fig. 4a) and phrenic nerve exhibited a normal appearance. However, in the sepsis group, 
a)

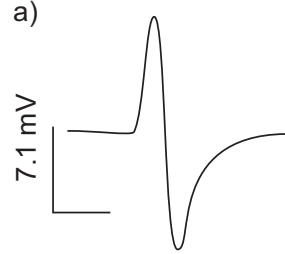

c)

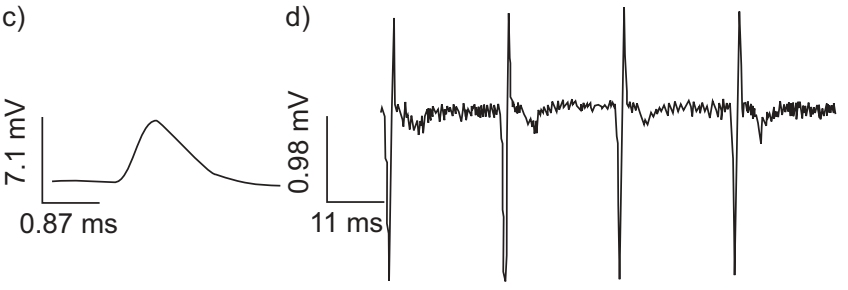

CMAP

b)

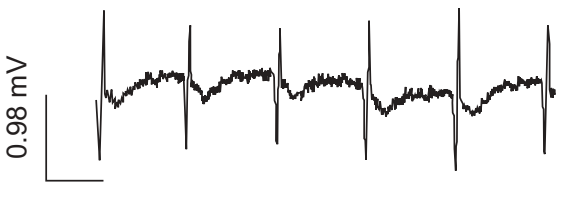

MUP

FIGURE 3. Morphology of motor unit action potential (MUP) recordings of the diaphragm muscle (sweep time $40 \mathrm{~ms}$ ) and compound action potential (CMAP) recordings of the phrenic nerve in the sham $(a, b)$ and sepsis $(c, d)$ groups on the 7 th day.

histopathological examination of the diaphragm showed accumulation of neutrophils and macrophages within the abdominal surface of diaphragm (fig. 4b). Clusters of inflammatory cells were present adhering to the wall of post-capillary venules and infiltrating the perivascular space of the muscle. The muscular fibres appeared normal (fig. 4c). Histopathological examination of the phrenic nerves revealed normal structure, except focally slight myelin degenerations, which characterised disruption and separation of the myelin lamella (fig. 5a). The intramuscular nerve fibres appeared normal (fig. 5b).

\section{DISCUSSION}

In the present study, sepsis induced electrophysiological abnormalities of the diaphragm in rats during a time-course study. Apparently, these electrophysiological abnormalities were present as early as $6 \mathrm{~h}$ after CLP procedure.

The CLP procedure was used to induce sepsis and investigate the electrophysiology of the diaphragm in these conditions. The CLP procedure, as well as lipopolysaccharide administration, is a commonly used model in the study of sepsis [15]. The CLP procedure involves a focal infection with more sustained exposure to the biological determinants of sepsis and may be closer to the clinical situation and, therefore, of greater relevance. In a previous experimental study, sepsis induced by either CLP procedure or lipopolysaccharide injection in rats elicited damage to the respiratory musculature [8].

Electrophysiological investigations, including needle electromyography of the diaphragm and phrenic nerve conduction studies, are useful to characterise and differentiate the neuropathical and myopathical involvement of the diaphragm. Here, needle electromyography of the diaphragm and the phrenic nerve conduction findings were consistent with phrenic nerve neuropathy. It was interesting to note that electrophysiology revealed no clear findings consistent with diaphragmatic myopathy. This is surprising, because it is generally found that sepsis also predisposes to myopathy. This can be explained by the similarity of their electophysiological features and the difficulty in separating the two entities.
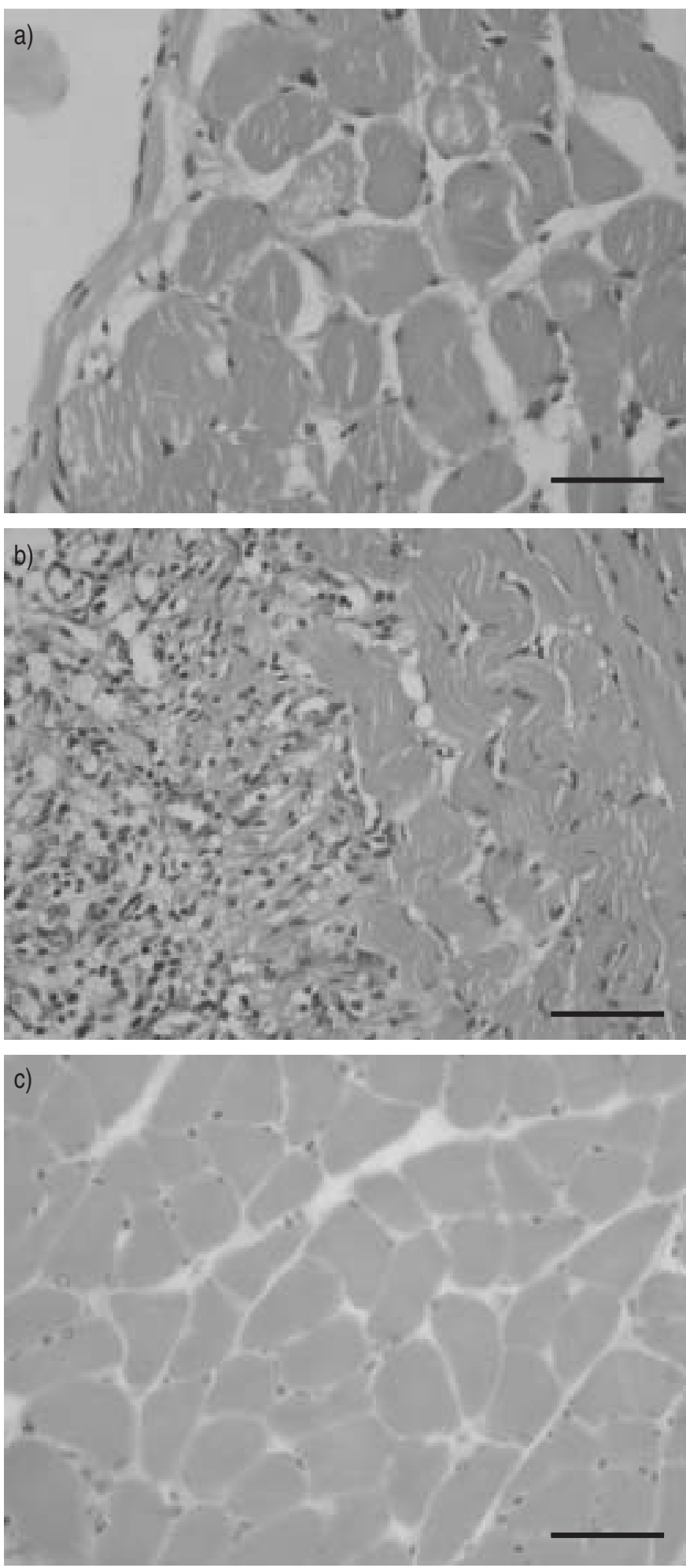

FIGURE 4. Histological slides showing: a) the normal appearance seen in the sham group; b) accumulation of neutrophils and macrophages on the abdominal surface of the diaphragm in the sepsis group; and c) normal structure of the diaphragm muscle in the sepsis group. Scale bar $=50 \mu \mathrm{m}$.

The electrophysiological findings presented here show different patterns of neuropathy, including axonal neuropathy and demyelinating neuropathy. An axonal neuropathy is 

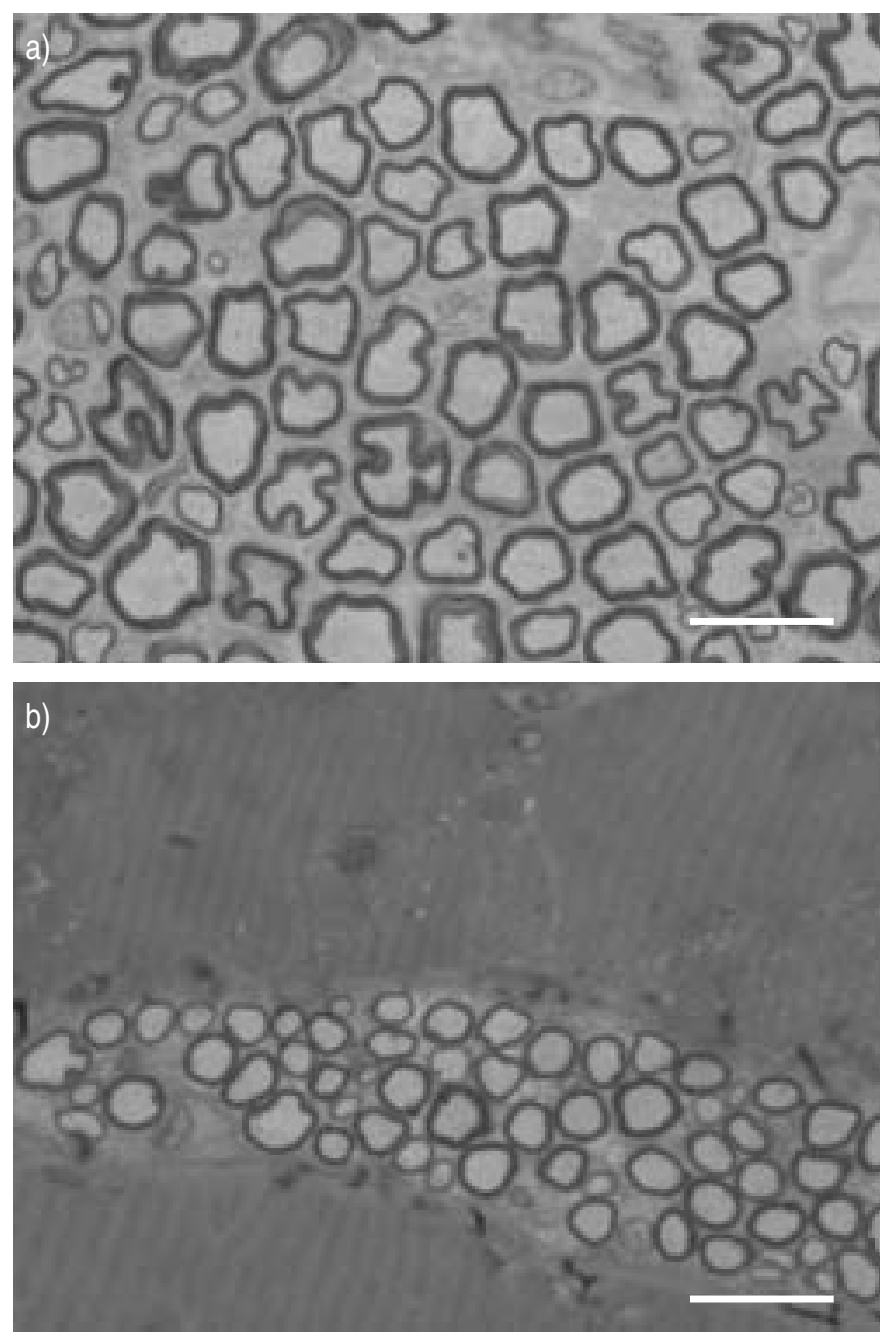

FIGURE 5. Histological slides showing: a) the phrenic nerve with focally slight myelin degenerations indicating disruption and separation of the myelin lamella in the sepsis group; and b) normal structure of the intramuscular nerve fibres. Scale bar $=20 \mu \mathrm{m}$.

characterised by the finding of preserved latency with diminished action potentials. In demyelinating neuropathy, the conduction time is prolonged while the action potential may be reduced or normal in amplitude [16]. The current findings show reduced CMAP amplitudes and scattered fibrillation potentials, indicating axonal changes of the phrenic nerve. In addition, the findings also show prolonged CMAP durations and latencies combined with reduced MUP numbers and recruitment patterns, indicating demyelinating changes of the phrenic nerve. Overall, these electrophysiological findings strongly suggest a phrenic nerve neuropathy during the timecourse of sepsis. Most strikingly, the electrophysiological abnormalities were present at $6 \mathrm{~h}$ and became worse at $24 \mathrm{~h}$ and on day 7. In fact, no data are available, in the current literature, which allows comparison between the present results and others performed in vivo in the diaphragm during the time-course of sepsis. However, the time-course found agrees with previous data reported by BOcZKOWSKI et al. [17]. These authors observed inflammatory cell infiltration at $6 \mathrm{~h}$ in the diaphragm, and diaphragmatic force reduction at $12 \mathrm{~h}$ after lipopolysaccharide inoculation, in a 48 -h time period.

Only a few studies have investigated the electrophysiology of the respiratory musculature in sepsis [18-21]. ZIFKO et al. [19] investigated the respiratory electrophysiology in ICU patients as part of critical illness polyneuropathy. ZIFKO et al. [19] found axonal degeneration and denervation of the phrenic nerve, and reported that diaphragmatic involvement was common in ICU [19]. ZOCHODNE et al. [20] studied respiratory electrophysiology in ICU patients, but the nature of the phrenic nerve neuropathy was not described in detail. WITT et al. [21] documented a reduction of the compound diaphragm action potential and primary axonal nerve degeneration. In these studies, most of the patients had sepsis either as a primary disease or as a complication during the course of their stay in ICU. However, it is generally accepted that the aetiology of neuropathy in critically ill patients is multifactorial and more than one factor may be responsible in any given patient. For instance, the neuromuscular blocking agents, steroids and antibiotics, which are used commonly in ICU, have also been implicated in inducing polyneuropathy or several types of myopathy [22]. In fact, it is difficult to compare the present results with the data published by these authors because the conditions of ICU patients are quite different and more complex. However, two lines of evidence are in agreement with the current findings. First, these studies primarily found phrenic nerve neuropathy. Secondly, the aetiological factor responsible for the neuropathy seemed to be sepsis. The present experimental sepsis model testified sepsis as the only aetiological factor. In this regard, the current findings reflect a true sepsis model inducing phrenic nerve neuropathy.

There has been much debate as to the incidence and nature of a myopathy, which may occur independently or in association with neuropathy in critically ill patients, and the role that electrophysiological testing and muscle biopsy may play in differentiating myopathy from neuropathy [19]. There is increasing evidence that myopathy may coexist with neuropathy [23-25]. BEDNARIK et al. [26] found significant overlapping of several pathogenic components of neuromuscular involvement, including axonal motor neuropathy, sensory neuropathy and myopathy in critically ill patients by electrophysiological and histological examinations. TROJABORG et al. [27] concluded that myopathy was much more common than polyneuropathy in critical illness. However, these studies have relied on electrophysiological measurements of limb nerve and muscle and have not utilised the techniques of needle electromyography of the diaphragm and phrenic nerve conduction study, which tests the respiratory system more precisely.

In order to ascertain whether the electrophysiological properties were associated with corresponding alterations in morphological signs of diaphragm muscle and phrenic nerve, histopathological examinations were also carried out. The muscle biopsy revealed no definite myopathical changes. However, the phrenic nerve biopsy showed focally slight myelin degenerations, indicating disruption and separation of the myelin lamella. Absence of a clear relationship between electrophysiological abnormalities and biopsy findings have also been reported by others [26-28]. Another possible explanation would be the fact that the present histopathological 
findings apply only to a single time point. It can be speculated that the histopathological abnormalities may not yet appear on day 7, as biopsies consistent with myopathy and/or neuropathy were taken during the 4-5th week of critical illness [2629].

In the present study, the rats became tachypnoeic and some had respiratory distress following CLP. The symptoms increased with time and caused respiratory arrest in some rats. As has been described in animal models, the work of breathing and energy demands of respiratory muscles remarkably increase in sepsis. Energy supply to the respiratory muscles, however, does not meet the demands. This can be explained by the reduction of cardiac output and blood pressure accompanying septic shock, and the inability of muscles to extract and use energy. Working under excessive inspiratory load may predispose to muscle fatigue or even muscle injury [8, 30, 31]. HUSSAIN et al. [32] studied the electromyography of the diaphragm and intercostal muscles, and concluded that respiratory failure in sepsis was due to the fatigue of the respiratory musculature. Whether high work load affects the diaphragm muscle was beyond the scope of this study, at least there were no definite myopathical changes.

In conclusion, the development of specific strategies or agents to decrease damage to respiratory musculature may possibly become a critical therapeutic modality in the prevention and treatment of respiratory failure. The current study showed phrenic nerve neuropathy during sepsis in rats. The study also showed how rapidly these neuropathical changes developed during the time-course study. This should be taken into consideration in the prevention and treatment of respiratory failure in septic patients.

\section{REFERENCES}

1 Montgomery AB, Stager MA, Carrico CJ, Hudson LD. Causes of mortality in patients with the adult respiratory distress syndrome. Am Rev Respir Dis 1985; 132: 485-489.

2 Bone RC, Balk RA, Cerra FB, et al. Definitions for sepsis and organ failure and guidelines for the use of innovative therapies in sepsis. Chest 1992; 101: 1644-1655.

3 Ebihara S, Hussain SN, Danialou G, Cho WK, Gottfried SB, Petrof BJ. Mechanical ventilation protects against diaphragm injury in sepsis: interaction of oxidative and mechanical stresses. Am J Respir Crit care Med 2002; 165: 221-228.

4 Kodama SI, Mikawa K, Nishina K, Maekawa N, Kagawa T, Obara H. Lidocaine attenuates sepsis-induced diaphragmatic dysfunction in hamsters. Crit Care Med 2000; 28: 2475-2479.

5 Krause KM, Moody MR, Andrade FH, et al. Peritonitis causes diaphragm weakness in rats. Am J Respir Crit Care Med 1998; 157: 1277-1282.

6 Fujimura N, Sumita S, Narimatsu E. Alteration in diaphragmatic contractility during septic peritonitis in rats: effect of polyethylene glycol-absorbed superoxide dismutase. Crit Care Med 2000; 28: 2406-2414.

7 Leon A, Boczkowski J, Dureuil B, Desmonts JM, Aubier M. Effects of endotoxic shock on diaphragmatic function in mechanically ventilated rats. J Appl Physiol 1992;72: 1466-1472.
8 Lin MC, Ebihara S, El Dwairi Q, et al. Diaphragm sarcolemmal injury is induced by sepsis and alleviated by nitric oxide synthase inhibition. Am J Respir Crit Care Med 1998; 158: 1656-1663.

9 Stephenson W. Deficiencies in the National Institute of Health's guidelines for the care and protection of laboratory animals. L Med Philos 1993; 18: 375-388.

10 Wichterman KA, Baue AE, Chaudry IH. Sepsis and septic shock: a review of laboratory models and a proposal. J Surg Res 1980; 29: 189-201.

11 Atis S, Comelekoglu U, Coskun B, Ozge A, Ersoz G, Talas D. Electrophysiological and histopathological evaluation of respiratory tract, diaphragm and phrenic nerve after dichlorvos inhalation in rats. Inhal Toxicol 2002; 14: 199-215.

12 MacLean IC, Mattioni TA. Phrenic nerve conduction studies: A new technique and its application in quadriplegic patients. Arch Phys Med Rehab 1991; 62: 70-72.

13 Terebuh BM, Johnson EW. The electrodiagnostic consultation including EMG examination. In: Johnson EW, Pease WS, eds. Practical Electromyography. 3rd Edn. Baltimore, USA, Williams \& Wilkins, 1997; pp. 1-13.

14 Aminoff $M$. Disturbances of other cranial nerves and phrenic nerve. In: Electomyography in Clinical Practice. 3rd Edn. New York, USA, Churchill Livingstone, 1998; pp. 525-532.

15 Piper RD, Cook DJ, Bone RC, Sibbald WJ. Introducing critical appraisal to studies of animal models investigating novel therapies in sepsis. Crit Care Med 1996; 24: 2059-2070.

16 Kimura J. Electrodiagnosis in disease of nerve and muscle: principles and practice. 2nd Edn. Philadelphia, USA, FA Davis, 1989.

17 Boczkowski J, Lanone S, Ungureanu-Longrois D, Danialou G, Fournier T, Aubier M. Induction of diaphragmatic nitric oxide synthase after endotoxin administration in rats: role on diaphragmatic contractile dysfunction. J Clin Invest 1996; 98: 1550-1559.

18 Polkey MI, Moxham J. Clinical aspects of respiratory muscle dysfunction in the critically ill. Chest 2001; 119: 926-939.

19 Zifko UA, Zipko HT, Bolton CF. Clinical and electrophysiological findings in critical illness polyneuropathy. $J$ Neurol Sci 1998; 159: 186-193.

20 Zochodne DW, Bolton CF, Wells GA, et al. Critical illness polyneuropathy. A complication of sepsis and multiple organ failure. Brain 1987; 110: 819-842.

21 Witt NJ, Zochodne DW, Bolton CF, et al. Peripheral nerve function in sepsis and multipl organ failure. Chest 1991; 99: 176-184.

22 Geller TJ, Kaiboriboon K, Fenton GA, Hayat GR. Vecuronium associated axonal motor neuropathy: a variant of critical illness polyneuropathy? Neuromuscul Discord 2001; 11: 579-582.

23 Bolton CF. Sepsis and systemic inflammatory response syndrome: neuromuscular manifestations. Crit Care Med 1996; 24: 1408-1416.

24 Lacomis D, Petrella JT, Giuliani MJ. Causes of neuromuscular weakness in the intensive care unit: a study of ninetytwo patients. Muscle Nerve 1998; 21: 610-617. 
25 Latronico N, Fenzi F, Recupero D, et al. Critical illness myopathy and neuropathy. Lancet 1996; 8: 1579-1582.

26 Bednarik J, Lukas Z, Vondracek P. Critical illness polyneuropathy: the electrophysiological components of a complex entity. Intensive Care Med 2003; 29: 1505-1514.

27 Trojaborg W, Weimer LH, Hays AP. Electrophysiological studies in critical illness associated weakness: myopathy or neuropathy - a reappraisal. Clin Neurophysiol 2001; 112: 1586-1593.

28 Coakley JH, Nagendran K, Yarwood GD, Honavar M, Hinds CJ. Patterns of neurophysiological abnormality in prolonged critical illness. Intensive Care Med 1998; 24: 801-807.

29 Roelofs RI. Critical illness polyneuropathy. Chest 1991; 99: 5-6.

30 Roussos C, Koutsoukou A. Respiratory failure. Eur Respir J 2003; 47: Suppl. 22, 3S-14S.

$31 \mathrm{McNeil}$ PL, Khakee R. Disruptions of muscle fiber plasma membranes: role in exercise induced damage. Am J Pathol 1992; 140: 1097-1109.

32 Hussain SN, Simkus G, Roussos C. Respiratory muscle fatigue: a cause of ventilatory failure in septic shock. $J$ Apply Physiol 1985; 58: 2033-2040. 